\title{
REMARKS ON THE INVERSE MEAN CURVATURE FLOW*
}

\author{
KNUT SMOCZYK ${ }^{\dagger}$
}

\begin{abstract}
In this short note we investigate the regularity of 2-surfaces evolving by its inverse mean curvature in an asymptotically flat Riemannian 3-manifold and derive an a-priori bound for the second fundamental form in terms of a quantity depending on the mean curvature and the elapsed time. This partially solves one of the 5 questions posed in [3]. The proof relies on the special geometry of asymptotically flat Riemannian manifolds and on the fact that the dimension of the evolving surface is 2 .
\end{abstract}

1. Introduction. Assume that $F_{t}: M^{n} \rightarrow N^{n+1}$ is a smooth family of immersions into a smooth Riemannian manifold $N$ evolving by the inverse mean curvature flow (IMCF)

$$
\frac{d}{d t} F=\frac{1}{H^{2}} \vec{H}
$$

where $\vec{H}$ is the outward pointing mean curvature vector and $H=|\vec{H}|$. In [3] the proof of the Penrose inequality [7] for a Riemannian 3-manifold $N^{3}$ of nonnegative scalar curvature has been announced. The proof is based on a weak formulation of the IMCF and will appear in [4]. The weak solution remains equal to the smooth solution until the latter ceases to exist or $M_{t}:=F_{t}\left(M^{n}\right)$ ceases to be a minimizing hull, which may happen sooner. In this paper we investigate the regularity of the classical solution of (1). From (4) (see below) and the maximum principle it easily follows that $H$ admits an upper bound that depends only on the initial immersion $F_{0}: M^{n} \rightarrow N^{n+1}$. A more important and delicate question is whether there exists an upper bound for the full second fundamental form $A$. Let us compare the situation to the mean curvature flow $\frac{d}{d t} F=-\vec{H}$. In [2] it was shown that a bound on the second fundamental form $A$ implies bounds on all covariant derivatives $\left|\nabla^{k} A\right|^{2}, \forall k \geq 0$ and consequently a smooth solution for the mean curvature flow exists as long as $A$ stays bounded. In special situations, as for example for mean convex solutions in the euclidean space, it is then possible to control the second fundamental form in terms of $H$, i.e. in terms of the speed alone. However, when working in arbitrary ambient spaces, this is no longer true. The aim of this paper is to derive similar results for the inverse mean curvature flow, i.e. we would like to control the second fundamental form and its covariant derivatives in terms of quantities that depend on the speed $\frac{1}{H}$. It turns out that we can do this, if we restrict the dimension to 2 . Interestingly enough, this is just the important case in general relativity and moreover we are able to bound $A$ in terms of the speed and the elapsed time when working in arbitrary asymptotically flat Riemannian manifolds $N$. Throughout this paper we will (somehow more generally) assume that $N$ is a complete Riemannian 3-manifold such that all curvature quantities and covariant derivatives of arbitrary order are bounded. Let us define two constants $a$ and $b$ by

$$
\begin{aligned}
a & :=\max _{M_{0}}|A|^{2} \\
b & :=\max _{M_{0}} H^{2} .
\end{aligned}
$$

*Received April 2, 1999; accepted for publication May 3, 1999.

${ }^{\dagger}$ Department of Mathematics, ETH-Zürich, Switzerland (ksmoczyk@math.ethz.ch). 
Then we will prove the following theorem:

Theorem 1.1. Assume that $n=2$. We can find constants $k, l \geq 0$ depending only on $a, b$ and on the ambient geometry such that

$$
|A|^{2} H^{2} \leq k+l t^{2}
$$

for all $t$, where a smooth solution of (1) exists. If $(0, T)$ is the maximal time interval for which a smooth solution of (1) exists, then

$$
\lim _{t \rightarrow T} \min _{M_{t}} H=0 .
$$

We summarize the evolution equations for the relevant geometric quantities in the following proposition.

Proposition 1.2.

$$
\begin{aligned}
& \frac{d}{d t} H=\frac{1}{H^{2}}\left\{\Delta H-\frac{2}{H}|\nabla H|^{2}-H\left(|A|^{2}+\overline{\operatorname{Ric}}(\nu, \nu)\right)\right\} \\
& \frac{d}{d t}|A|^{2}=\frac{1}{H^{2}}\left\{\Delta|A|^{2}-2|\nabla A|^{2}-\frac{4}{H}\left\langle h_{i j}, \nabla_{i} H \nabla_{j} H\right\rangle-4 H \operatorname{trA}^{3}+2|\mathrm{~A}|^{4}\right. \\
& +2|A|^{2} \overline{\operatorname{Ric}}(\nu, \nu)-4 H\left\langle h_{i j}, \bar{R}_{0 i 0 j}\right\rangle-4 h^{i j}\left(h_{j l} \bar{R}_{m i}^{l}{ }_{m i}^{m}-h^{l m} \bar{R}_{l i m j}\right) \\
& \left.-2 h^{i j}\left(\bar{\nabla}_{j} \bar{R}_{0 l i}{ }^{l}+\bar{\nabla}_{l} \bar{R}_{0 i j}{ }^{l}\right)\right\}
\end{aligned}
$$

LEMMA 1.3. If $n=2$ then we can find constants $c_{1}, c_{2} \geq 0$, depending only on the geometry of $N^{3}$, such that

$$
\begin{aligned}
\frac{d}{d t}|A|^{2} & \leq \frac{1}{H^{2}}\left\{\Delta|A|^{2}-2|\nabla A|^{2}-\frac{4}{H}\left\langle h_{i j}, \nabla_{i} H \nabla_{j} H\right\rangle-2 H^{2}\left(3|A|^{2}-H^{2}\right)\right. \\
& \left.+2|A|^{2}\left(|A|^{2}+\overline{\operatorname{Ric}}(\nu, \nu)\right)+c_{1}|A|^{2}+c_{2}\right\}
\end{aligned}
$$

Proof. Since $n=2$ we make the observation that

$$
\operatorname{tr} \mathrm{A}^{3}=\lambda_{1}^{3}+\lambda_{2}^{3}=\left(\lambda_{1}+\lambda_{2}\right)\left(\lambda_{1}^{2}-\lambda_{1} \lambda_{2}+\lambda_{2}^{2}\right)=\frac{1}{2} \mathrm{H}\left(3|\mathrm{~A}|^{2}-\mathrm{H}^{2}\right),
$$

where $\lambda_{1}, \lambda_{2}$ denote the two principal curvatures of $M$. Since all curvature quantities on $N$ are bounded we can repeatedly apply Schwarz' inequality to bound each term in the evolution equation (5) that involves ambient curvature quantities by a constant multiple of $|A|^{2}$. This proves the lemma.

REMARK. We want to point out that the following calculations strongly depend on the identity (7) and that in higher dimensions the bad term $H \operatorname{tr} \mathrm{A}^{3}$ cannot be reduced to a term that seems to be good enough to imply bounds on derivatives of $A$ in terms of $H$.

LEMMA 1.4. If $n=2$ then we can find constants $c_{3}, c_{4} \geq 0$, depending only on the ambient geometry, such that

$$
\begin{aligned}
\frac{d}{d t}\left(|A|^{2} H^{2}\right) & \leq \frac{1}{H^{2}}\left\{\Delta\left(|A|^{2} H^{2}\right)-2\left\langle\nabla|A|^{2}, \nabla H^{2}\right\rangle-6|A|^{2}|\nabla H|^{2}-2 H^{2}|\nabla A|^{2}\right. \\
& \left.-4\left\langle h_{i j}, \nabla_{i} H \nabla_{j} H\right\rangle-2 H^{4}\left(3|A|^{2}-H^{2}\right)+c_{3}|A|^{2} H^{2}+c_{4} H^{2}\right\}
\end{aligned}
$$


Proof. This follows directly from Lemma 1.3, the evolution equation for $H$ and the bound for $\overline{\operatorname{Ric}}(\nu, \nu)$.

Now let $d \geq 0$ be a constant to be chosen later and set

$$
f_{d}:=|A|^{2} H^{2}+\left(\frac{c_{3}}{2}+d\right) H^{2}
$$

LEMMA 1.5. If $n=2$ then we can find a constant $c_{5} \geq 0$, depending only on the ambient geometry, such that for any $d \geq 0$ we obtain the inequality

$$
\begin{aligned}
\frac{d}{d t} f_{d} & \leq \frac{1}{H^{2}}\left\{\Delta f_{d}-\frac{2}{H}\left\langle\nabla H, \nabla f_{d}\right\rangle+4|A| H|\nabla H|^{2}-\left(c_{3}+2 d\right)|\nabla H|^{2}\right\} \\
& -2|A|^{2} H^{2}+c_{4}+d c_{5} .
\end{aligned}
$$

Proof. Using Lemma 1.4 and the evolution equation for $H$ we obtain

$$
\begin{aligned}
\frac{d}{d t} f_{d} & \leq \frac{1}{H^{2}}\left\{\Delta f_{d}-4 H\left\langle\nabla|A|^{2}, \nabla H\right\rangle-6|A|^{2}|\nabla H|^{2}-2 H^{2}|\nabla A|^{2}\right. \\
& \left.-4 H\left\langle h_{i j}, \nabla_{i} H \nabla_{j} H\right\rangle-6\left(\frac{c_{3}}{2}+d\right)|\nabla H|^{2}\right\} \\
& -2 H^{2}\left(3|A|^{2}-H^{2}\right)+c_{4}-2 d \overline{\operatorname{Ric}}(\nu, \nu)
\end{aligned}
$$

Now let

$$
Q^{2}:=\left|\nabla_{i} h_{k l} H+\nabla_{i} H h_{k l}\right|^{2}=H^{2}|\nabla A|^{2}+|A|^{2}|\nabla H|^{2}+H\left\langle\nabla|A|^{2}, \nabla H\right\rangle .
$$

Then it follows that

$$
\begin{aligned}
& -4 H\left\langle\nabla|A|^{2}, \nabla H\right\rangle-6|A|^{2}|\nabla H|^{2}-2 H^{2}|\nabla A|^{2}-4 H\left\langle h_{i j}, \nabla_{i} H \nabla_{j} H\right\rangle \\
& -6\left(\frac{c_{3}}{2}+d\right)|\nabla H|^{2} \\
& =-2 H\left\langle\nabla|A|^{2}, \nabla H\right\rangle-4|A|^{2}|\nabla H|^{2}-2 Q^{2}-4 H\left\langle h_{i j}, \nabla_{i} H \nabla_{j} H\right\rangle \\
& -6\left(\frac{c_{3}}{2}+d\right)|\nabla H|^{2} \\
& =-\frac{2}{H}\left\langle\nabla H, \nabla f_{d}\right\rangle-2 Q^{2}-4 H\left\langle h_{i j}, \nabla_{i} H \nabla_{j} H\right\rangle-\left(c_{3}+2 d\right)|\nabla H|^{2}
\end{aligned}
$$

and the result follows from

$$
\begin{aligned}
2|A|^{2} & \geq H^{2} \\
-4 H\left\langle h_{i j}, \nabla_{i} H \nabla_{j} H\right\rangle & \leq 4|A| H|\nabla H|^{2} \\
-2 d \overline{\operatorname{Ric}}(\nu, \nu) & \leq d c_{5} .
\end{aligned}
$$

Next we define

$$
h_{d}:=f_{d}-\left(c_{4}+d c_{5}\right) t-k_{d}
$$

where

$$
k_{d}:=\left(a+\frac{c_{3}}{2}+d\right) b
$$


We come to the proof of Theorem 1.1.

Proof. Fix a time interval $[0, \tau)$, where a smooth solution of the IMCF exists. We first prove that we can find a constant $d$ such that $h_{d} \leq 0$ for all $t<\tau$. This is a consequence of the parabolic maximum principle, if we can show that $\frac{d}{d t} h_{d} \leq 0$ at all points where $\Delta h_{d} \leq 0, \nabla h_{d}=0, h_{d}=0$. But at such a point we can use (10) to conclude

$$
\frac{d}{d t} h_{d} \leq\left(4|A| H-c_{3}-2 d\right) \frac{|\nabla H|^{2}}{H^{2}}-2|A|^{2} H^{2}
$$

If $h_{d}=0$ we obtain

$$
(16)|A|^{2} H^{2} \leq\left(c_{4}+d c_{5}\right) t+k_{d} \leq\left(c_{4}+c_{5} d\right) \tau+k_{d}=c_{4} \tau+b\left(\frac{c_{3}}{2}+a\right)+d\left(b+c_{5} \tau\right) .
$$

Let

$$
\begin{aligned}
& \widetilde{A}:=\max \left\{c_{4}, c_{5}\right\} \\
& \widetilde{B}:=\max \left\{b\left(\frac{c_{3}}{2}+a\right), b, 1\right\}
\end{aligned}
$$

Then

$$
\begin{aligned}
& 4 \sqrt{c_{4} \tau+b\left(\frac{c_{3}}{2}+a\right)+d\left(b+c_{5} \tau\right)}-c_{3}-2 d \\
& \leq 2(2 \sqrt{\widetilde{A} \tau+\widetilde{B}+d(\widetilde{B}+\widetilde{A} \tau)}-d) \\
& =2(2 \sqrt{(d+1)(\widetilde{A} \tau+\widetilde{B})}-d) .
\end{aligned}
$$

Provided $d \geq 1$, this is smaller than

$$
2(2 \sqrt{2 d(\tilde{A} \tau+\widetilde{B})}-d)=2(\sqrt{d} \sqrt{A \tau+B}-d)
$$

where we have set

$$
\begin{aligned}
& A:=8 \widetilde{A} \\
& B:=8 \widetilde{B} .
\end{aligned}
$$

If we choose $d:=A \tau+B$ the last term vanishes and consequently

$$
4 \sqrt{c_{4} \tau+b\left(\frac{c_{3}}{2}+a\right)+d\left(b+c_{5} \tau\right)}-c_{3}-2 d \leq 0 .
$$

We summarize: On any fixed time interval $[0, \tau)$, where a smooth solution of the IMCF exists we can find constants $A, B$ depending only on $a, b$ and the ambient geometry, such that

$$
h_{A \tau+B} \leq 0 \text { on }[0, \tau) .
$$

This means that

$$
\begin{aligned}
|A|^{2} H^{2} & \leq\left(c_{4}+d c_{5}\right) t+k_{d} \\
& =\left(c_{4}+(A \tau+B) c_{5}\right) t+\left(a+\frac{c_{3}}{2}+(A \tau+B)\right) b \\
& \leq A c_{5} \tau^{2}+\left(c_{4}+B c_{5}+A b\right) \tau+\left(a+\frac{c_{3}}{2}+B\right) b \\
& =\epsilon_{1} \tau^{2}+\epsilon_{2} \tau+\epsilon_{3}
\end{aligned}
$$


where

$$
\begin{aligned}
& \epsilon_{1}:=A c_{5} \\
& \epsilon_{2}:=c_{4}+B c_{5}+A b \\
& \epsilon_{3}:=\left(a+\frac{c_{3}}{2}+B\right) b .
\end{aligned}
$$

Finally we use $\epsilon_{2} \tau \leq \frac{\epsilon_{2}^{2}}{2} \tau^{2}+\frac{1}{2}$ to conclude

$$
|A|^{2} H^{2} \leq k+l \tau^{2}
$$

with two constants $k$ and $l$ depending only on $a, b$ and on the ambient geometry but not on $\tau$. Since $\tau$ was arbitrary we are done with the first part of Theorem 1.1. This estimate states that $|A|^{2}$ is bounded as long as $H$ is bounded away from zero. Once we have a bound on the full second fundamental form we can use results of Krylov [6] (see also [1] and [2] for similar arguments) to derive bounds on all derivatives of the second fundamental form. This proves that a smooth solution must exists as long as $H$ is positive.

\section{REFERENCES}

[1] C. Gerhardt, Flow of nonconvex hypersurfaces into spheres, J. Diff. Geom., 32 (1990), pp. 299-314.

[2] G. HuISKen, Contracting convex hypersurfaces in Riemannian manifolds by their mean curvature, Invent. Math., 84 (1986), pp. 463-480.

[3] G. Huisken And T. Ilmanen, The Riemannian Pentose Inequality, in IMRN 20, 1997, pp. 1045-1058.

[4] G. Huisken AND T. Ilmanen, The inverse mean curvature flow and the Riemannian Penrose inequality, MPI-Leipzig preprint No. 16.

[5] G. Huisken and T. Ilmanen, A note on the inverse mean curvature flow, preprint, 1998.

[6] N. V. KRYLov, Nonlinear Elliptic and Parabolic Equations of the Second Order, Reidel, Dordrecht, 1987.

[7] R. Penrose, Naked Singularities, Ann. New York Acad. Sci., 224 (1973), pp. 125-134. 
K. SMOCZYK 